\title{
CONSTRUCTION OF VECTOR FIELDS AND RICCATI FOLIATIONS ASSOCIATED TO GROUPS OF PROJECTIVE AUTOMORPHISMS
}

\author{
FABIO SANTOS AND BRUNO SCÁRDUA
}

\begin{abstract}
Our main result states that given a finitely generated subgroup $G$ of Aut $(\mathbb{C} P(2))$, there is an algebraic foliation $\mathcal{F}$ on a complex projective 3-manifold $M^{3}$ with a bundle structure over $\mathbb{C} P(1)$ and fiber $\mathbb{C} P(2)$, such that $\mathcal{F}$ is transverse to almost every fiber of the bundle and with global holonomy conjugate to $G$.
\end{abstract}

\section{INTRODUCTION}

Foliations transverse to fibrations are among the simplest (constructive) examples of foliated manifolds, once regarded as suspensions of groups of diffeomorphisms (see [1] and [2]). Thus one expects to perform a nice study of them in the global theoretic aspect. In the complex algebraic setting, foliations usually exhibit singularities so this possibility cannot be excluded. Very representative examples of either situation are given by the class of Riccati foliations (see [5]) in dimension two. A very interesting study is performed in [7] and a complete reference on the two dimensional case is [6]. In this paper we study one-dimensional holomorphic foliations with singularities which are transverse to a given holomorphic fibration outside a certain subset called the exceptional set. Let us first recall the classical notion. Let $\eta=(E, \pi, B, F)$ be a fibre bundle with total space $E$, fiber $F$, basis $B$ and projection $\pi: E \rightarrow B$. A foliation $\mathcal{F}$ on $E$ is transverse to $\eta$ if:

(1) for each $p \in E$, the leaf $L_{p}$ of $\mathcal{F}$ with $p \in L_{p}$ is transverse to the fiber $\pi^{-1}(q)$, $q=\pi(p)$

(2) $\operatorname{dim}(\mathcal{F})+\operatorname{dim}(F)=\operatorname{dim}(E)$; and

(3) for each leaf $L$ of $\mathcal{F}$, the restriction $\left.\pi\right|_{L}: L \rightarrow B$ is a covering map. According to Ehresmann (see [2]) if the fiber $F$ is compact, then the conditions (1) and (2) already imply (3). In the complex setting, all the objects above are holomorphic by hypothesis and several are the interesting frameworks (see [9] for the complex hyperbolic case and [8] for the codimension one case). Under the presence of singularities, a weaker notion must be introduced. We shall say that $\mathcal{F}$ is transverse to almost every fiber of the fibre bundle $\eta$ if there is an analytic subset $\Lambda(\mathcal{F}) \subset E$ which is the union of fibers of $\eta$, such that the restriction $\mathcal{F}_{0}$ of $\mathcal{F}$ to $E_{0}=E \backslash \Lambda$ is transverse to the natural subbundle $\eta_{0}$ of $\eta$ having $E_{0}$ as total space. If $\Lambda(\mathcal{F})$ is minimal with this property, then $\Lambda(\mathcal{F})$ is called the exceptional set of $\mathcal{F}$. By

Received by the editors August 27, 2009.

2010 Mathematics Subject Classification. Primary 37F75, 32S65; Secondary 32M25, $32 \mathrm{M} 05$.

Key words and phrases. Holomorphic foliation, holonomy, projective automorphism.

(C)2010 American Mathematical Society 
a Riccati foliation we mean a foliation $\mathcal{F}$ as above, for which the exceptional set $\Lambda(\mathcal{F})$ is $\mathcal{F}$-invariant. In particular, we shall consider the global holonomy of $\mathcal{F}$ as the global holonomy of the restriction $\mathcal{F}_{0}$ on $E_{0}=E \backslash \Lambda(\mathcal{F})$. In the classical situation of Riccati foliations in $\overline{\mathbb{C}} \times \overline{\mathbb{C}}$, the global holonomy is a finitely generated group of Möbius transformations, i.e., a finitely generated subgroup of $\operatorname{PSL}(2, \mathbb{C})$. In [5] the author proves that given a finitely generated subgroup $G<P S L(2, \mathbb{C})$ there is a Riccati foliation in $\overline{\mathbb{C}} \times \overline{\mathbb{C}}$ for which the global holonomy is conjugated to $G$. In this work we consider the problem of synthesis of Riccati foliations on $\overline{\mathbb{C}} \times \mathbb{C} P(2)$. Our main result below may apply to the study of (singular) projective foliations with homogeneous transverse structure (see [2]) in codimension $\geq 2$.

Theorem 1.1 (Synthesis theorem). Let $f_{1}, \ldots, f_{r} \in \operatorname{Aut}(\mathbb{C} P(2))$ be holomorphic diffeomorphisms of $\mathbb{C} P(2)$ and let $x_{0}^{0}, x_{1}^{0}, \ldots, x_{r}^{0} \in \overline{\mathbb{C}}$ be $r+1$ points in $\mathbb{C} P(2)$. There is an algebraic (Riccati) foliation $\mathcal{F}$ on a complex projective 3 -manifold $M^{3}$, admitting a holomorphic fibre bundle structure $\eta=\left(M^{3}, \pi, \overline{\mathbb{C}}, \mathbb{C} P(2)\right)$, over $\mathbb{C} P(2)$ with fiber $\overline{\mathbb{C}}$, with the following properties:

(1) $\mathcal{F}$ is transverse to almost every fiber of $\eta$.

(2) The invariant fibers of $\mathcal{F}$ are $\pi^{-1}\left(x_{0}^{0}\right), \ldots, \pi^{-1}\left(x_{r}^{0}\right)$.

(3) The global holonomy of $\mathcal{F}$ is conjugate to the subgroup of $\operatorname{Aut}(\mathbb{C} P(2))$ generated by $f_{1}, \ldots, f_{r}$.

\section{Fixed points of automorphisms of $\mathbb{C} P(2)$}

The group of automorphisms of $\mathbb{C} P(n)$ is induced by the general linear group; that is, $\operatorname{Aut}(\mathbb{C} P(n)) \cong \operatorname{PGL}(n+1, \mathbb{C})$ (see [3]), it identifies an isomorphism $T: \mathbb{C}^{n+1}$ $\rightarrow \mathbb{C}^{n+1}$ with the biholomorphism of the complex projective space $[T]$ defined by: if $r \subset \mathbb{C}^{n+1}$ is a complex line containing $0 \in \mathbb{C}^{n+1}$, then $s=T(r)$ is a complex line containing $0 \in \mathbb{C}^{n+1}$ and we consider $[T]: \mathbb{C} P(n) \rightarrow \mathbb{C} P(n)$ given by $[T](r \backslash\{0\})=s \backslash\{0\}$.

Aiming the study of Riccati foliations on $\overline{\mathbb{C}} \times \mathbb{C} P(2)$ through the global holonomy we perform the classification of holomorphic diffeomorphisms of $\mathbb{C} P(2)$ by the set of fixed points. Indeed, we use the Jordan canonical forms to obtain the following.

Proposition 2.1. If $f: \mathbb{C} P(2) \rightarrow \mathbb{C} P(2)$ is a biholomorphism and $\Sigma(f) \subset \mathbb{C} P(2)$ denotes its set of fixed points, then we have the following six possibilities:

(1) $\Sigma(f)$ is a set of one, two or three points.

(2) $\Sigma(f)$ consists of a projective line.

(3) $\Sigma(f)$ consists of one projective line plus one point.

(4) $\Sigma(f)$ has dimension two and $\Sigma(f)=\mathbb{C} P(2)$.

In particular, $f$ is conjugate in $\operatorname{Aut}(\mathbb{C} P(2))$ to a map $g \in \operatorname{Aut}(\mathbb{C} P(2))$ of the form,

$$
\begin{array}{ll}
g(x: y: z)=\left(\lambda_{0} x+y: \lambda_{0} y+z: \lambda_{0} z\right), & g(x: y: z)=\left(\lambda_{0} x+y: \lambda_{0} y: \lambda_{1} z\right), \\
g(x: y: z)=\left(\lambda_{0} x: \lambda_{1} y: \lambda_{2} z\right), & g(x: y: z)=\left(\lambda_{0} x: \lambda_{0} y: \lambda_{1} z\right), \\
g(x: y: z)=\left(\lambda_{0} x+y: \lambda_{0} y: \lambda_{0} z\right), & g(x: y: z)=(x: y: z),
\end{array}
$$

where $\lambda_{0}, \lambda_{1}, \lambda_{2} \in \mathbb{C} \backslash\{0\}$, respectively. 
Motivated by Proposition 2.1, we introduce the following auxiliary definition.

Definition 2.2. We shall say that a biholomorphism $f: \mathbb{C} P(2) \rightarrow \mathbb{C} P(2)$ different from the identity is:

(i) of type $P k$ with $k \in\{1,2,3\}$, if $\Sigma(f)$ consists of $k$ points,

(ii) of type $S L 1$, if $\Sigma(f)$ consists of one projective line,

(iii) of type $P 1 S L 1$ if $\Sigma(f)$ consists of one point and one projective line.

\section{Construction of foliations, proof of the Synthesis theorem}

In this section we prove Theorem 1.1. We proceed as in [5]; nevertheless, some differences and difficulties arise.

Proof of Theorem [1.1. Let $x_{0}^{0}=0, x_{1}^{0}, \ldots, x_{r}^{0}$ be points in $\mathbb{C}$. For each $j \in\{0,1, \ldots, r\}$ we take $D_{j}$ to be a disk of center $x_{j}^{0}$ and radius $\rho>0$ such that $\left|x_{j}^{0}-x_{k}^{0}\right|>2 \rho$ for all $j \neq k, 0 \leq j, k \leq r$. For each $j \in\{1, \ldots, r\}$ we choose $x_{j}^{\prime}=x_{j}^{0}+\frac{\rho}{2} \in D_{j} \backslash\left\{x_{j}^{0}\right\}$ and $x_{j}^{\prime \prime}=\frac{\rho}{2} \exp \left(\frac{2 \pi \sqrt{-1}(j-1)}{r}\right) \in D_{0} \backslash\{0\}$. For each $j \in\{1, \ldots, r\}$ we take $\alpha_{j}:[0,1] \rightarrow \mathbb{C}$ to be simple curves such that:

(i) $\alpha_{j}(0)=x_{j}^{\prime \prime}$ and $\alpha_{j}(1)=x_{j}^{\prime}$;

(ii) $\alpha_{j}([0,1]) \cap D_{k}=\emptyset$ if $j \neq k, k \neq 0$;

(iii) $\alpha_{j}([0,1]) \cap \alpha_{k}([0,1])=\emptyset$ if $j \neq k$;

(iv) $\alpha_{j}([0,1]) \cap D_{0}$ and $\alpha_{j}([0,1]) \cap D_{j}$ are segments of straight lines contained in diameters of $D_{0}$ and $D_{j}$ respectively.

Let $A_{1}, \ldots, A_{r}$ be strips around $\alpha_{1}, \ldots, \alpha_{r}$, respectively, such that:

(v) $A_{j} \cap D_{k}=\emptyset$ if $j \neq k, k \neq 0$;

(vi) $A_{j} \cap A_{k}=\emptyset$ if $j \neq k$;

(vii) $A_{j} \cap D_{0}$ and $A_{j} \cap D_{j}$ are contained in sectors of $D_{0}$ and $D_{j}, 1 \leq j \leq r$, respectively.

Let $U=\left(\bigcup_{j=1}^{r} A_{j}\right) \cup\left(\bigcup_{j=0}^{r} D_{j}\right)$ be a set and let $\gamma=\partial U$ be a simple curve. Let $T$ be a tubular neighborhood of $\gamma$ and let $V=(\overline{\mathbb{C}} \backslash U) \cup T$ be a set. Then $\left\{A_{1}, \ldots, A_{r}, D_{0}, \ldots, D_{r}, V\right\}$ is a covering of $\overline{\mathbb{C}}$ by open sets. For every $k \in\{1, \ldots, r\}$ we consider affine coordinates $\left(x, \widetilde{y}_{k}, \widetilde{z}_{k}\right),\left(x, \widetilde{u}_{k}=\frac{1}{\widetilde{y}_{k}}, \widetilde{v}_{k}=\frac{\widetilde{z}_{k}}{\widetilde{y}_{k}}\right),\left(x, \widetilde{s}_{k}=\frac{1}{\widetilde{z}_{k}}, \widetilde{t}_{k}=\right.$ $\left.\frac{\widetilde{y}_{k}}{\widetilde{z}_{k}}\right)$ in $A_{k} \times \mathbb{C}^{2} \hookrightarrow A_{k} \times \mathbb{C} P(2)$, and for each $j \in\{0,1, \ldots, r\}$ we consider affine coordinates $\left(x, y_{j}, z_{j}\right),\left(x, u_{j}=\frac{1}{y_{j}}, v_{j}=\frac{z_{j}}{y_{j}}\right),\left(x, s_{j}=\frac{1}{z_{j}}, t_{j}=\frac{y_{j}}{z_{j}}\right)$ in $D_{j} \times \mathbb{C}^{2}$. Put affine coordinates $(w, y, z)$ in $V \times \mathbb{C}^{2}$ where $w=\frac{1}{x} \in V$ and $(y, z) \in \mathbb{C}^{2}$.

We take in each set of the form $A_{k} \times \mathbb{C} P(2), V \times \mathbb{C} P(2)$ and $D_{j} \times \mathbb{C} P(2)$ a local model of foliation and glue them together. The local models are as follows:

(1) In $A_{k} \times \mathbb{C P}(2)$ we consider the horizontal foliation whose leaves are of the form $A_{k} \times\{p\}, p \in \mathbb{C} P(2)$ for each $j \in\{1, \ldots, r\}$.

(2) In $V \times \mathbb{C} P(2)$ we consider the horizontal foliation whose leaves are of the form $V \times\{p\}, p \in \mathbb{C} P(2)$.

(3) In $D_{j} \times \mathbb{C}^{2}$ we consider the singular holomorphic foliation $\mathcal{F}_{j}$ induced by the vector field $X_{j}$ in $D_{j} \times \mathbb{C}^{2}$ for every $j \in\{0,1, \ldots, r\}$. In fact, for each $j \in\{0,1, \ldots, r\}$ we consider $f_{j}$ the global holonomy of $\mathcal{F}_{j}$ on $D_{j} \times \mathbb{C} P(2)$, where $f_{0}=\left(f_{1} \circ \cdots \circ f_{r}\right)^{-1}$. In this case we note that (Proposition 2.1): for each $j$ there exists an affine coordinate such that it identifies $f_{j}$ with the 
map $F_{j}: \mathbb{C}^{2} \rightarrow \mathbb{C}^{2}$ and $F_{j}$ can be written in one of the following forms:

(a) $F_{j}(u, v)=(u+\mu v, v+\mu)$ if $f_{j}$ is of type P1.

(b) $F_{j}(u, v)=(\mu u+\nu v, \mu v)$ if $f_{j}$ is of type $\mathrm{P} 2$.

(c) $F_{j}(u, v)=\left(\lambda^{\prime} u, \lambda^{\prime \prime} v\right)$ if $f_{j}$ is of type P3.

(d) $F_{j}(u, v)=\left(\lambda^{\prime \prime} u, \lambda^{\prime \prime} v\right)$ if $f_{j}$ is SL1.

(e) $F_{j}(u, v)=(u+\nu v, v)$ if $f_{j}$ is of type P1SL1, where $\lambda^{\prime}, \lambda^{\prime \prime}, \mu, \nu \in \mathbb{C} \backslash\{0\}$ are different.

- In case (c) or case (d), we consider the singular holomorphic foliation $\mathcal{F}_{j}$ on $D_{j} \times \mathbb{C}^{2}$ given by the vector field

$$
X_{j}\left(x, y_{j}, z_{j}\right)=\left(x-x_{j}^{0}\right) \frac{\partial}{\partial x}+\alpha_{j}^{\prime} y_{j} \frac{\partial}{\partial y_{j}}+\alpha_{j}^{\prime \prime} z_{j} \frac{\partial}{\partial z_{j}}
$$

where $\exp \left(2 \pi \sqrt{-1} \alpha_{j}^{\prime}\right)=\lambda_{j}^{\prime}$ and $\exp \left(2 \pi \sqrt{-1} \alpha_{j}^{\prime \prime}\right)=\lambda_{j}^{\prime \prime}$. However, in case (d) we also have $\alpha_{j}^{\prime \prime}=\alpha_{j}^{\prime}$ and $\lambda_{j}^{\prime \prime}=\lambda_{j}^{\prime}$. Let $\gamma_{j}(\theta)=\left(x_{j}^{0}+\rho_{j} \exp (\sqrt{-1} \theta), 0,0\right), 0 \leq \theta \leq 2 \pi$ be a curve where $0<\rho_{j}<\rho$. Let $\Sigma_{j}=\left\{p_{j}\right\} \times \mathbb{C}^{2}, p_{j} \in \gamma_{j}([0,2 \pi])$.

Claim 3.1. The holonomy transformation of $\mathcal{F}_{j}$ associated to $\Sigma_{j}$ and $\gamma_{j}$ is of the form $\left(y_{j}, z_{j}\right) \mapsto\left(\lambda_{j}^{\prime} y_{j}, \lambda_{j}^{\prime \prime} z_{j}\right)$ where the foliation $\mathcal{F}_{j}$ on $D_{j} \times \mathbb{C}^{2}$ is induced by $X_{j}$ (equation (3.1)).

In fact, suppose $\Sigma_{j}=\left\{x_{j}^{0}+\rho_{j}\right\} \times \mathbb{C}^{2}$, a local transverse section, and $p_{j}=$ $\left(x_{j}^{0}+\rho_{j}, 0,0\right) \in \Sigma_{j}$. Suppose $\pi_{1}: D_{j} \times \mathbb{C}^{2} \rightarrow D_{j}, \pi_{1}(x, y, z)=x$. Observe that the fibers $\pi_{1}^{-1}(x), x \neq x_{j}^{0}$, are transverse to $\mathcal{F}_{j}$. We consider $q=\left(x_{j}^{0}+\rho_{j}, y_{j}, z_{j}\right) \in \Sigma_{j}$ and $\gamma_{q}(\theta)=\left(x(\theta), y_{j}(\theta), z_{j}(\theta)\right)$ the lifting of $\gamma_{j}$ by $\pi_{1}$ with base point $q$. Therefore $x^{\prime}(\theta)=\pi_{1}\left(\gamma_{q}^{\prime}(\theta)\right)=\pi_{1}\left(\gamma_{j}^{\prime}(\theta)\right)=\sqrt{-1} \rho_{j} \exp (\sqrt{-1} \theta)$, and, if $Y_{j}=\left(y_{j}, z_{j}\right) \in \mathbb{C}^{2}$, then

$$
\frac{Y_{j}^{\prime}}{x^{\prime}}=\frac{Y_{j}^{\prime}}{\sqrt{-1} \rho_{j} \exp (\sqrt{-1} \theta)} .
$$

On the other hand, by equation (3.1) we have $\frac{d x}{d T}=x-x_{j}^{0}$ and

$$
\frac{d Y_{j}}{d T}=\left[\begin{array}{cc}
\alpha_{j}^{\prime} & 0 \\
0 & \alpha_{j}^{\prime \prime}
\end{array}\right] \cdot\left[\begin{array}{l}
y_{j} \\
z_{j}
\end{array}\right]=A Y_{j}
$$

so we obtain

$$
\frac{d Y_{j}}{d x}=\frac{d Y_{j}}{d T} \cdot \frac{d T}{d x}=\frac{\frac{d Y_{j}}{d T}}{\frac{d x}{d T}}=\frac{A Y_{j}}{x-x_{j}^{0}}
$$

and we have

$$
\frac{Y_{j}^{\prime}}{\sqrt{-1} \rho_{j} \exp (\sqrt{-1} \theta)}=\frac{Y_{j}^{\prime}}{x^{\prime}}=\frac{A Y_{j}}{\rho_{j} \exp (\sqrt{-1} \theta)},
$$


then $Y_{j}^{\prime}=\sqrt{-1} A Y_{j}$. Notice that the solution of last equation such that $Y_{j}(0)=$ $\left(y_{j}, z_{j}\right)$ is $Y_{j}(\theta)=\exp (\sqrt{-1} \theta A) \cdot Y_{j}(0)$. Therefore the holonomy is the biholomorphism $f: \Sigma_{j} \rightarrow \Sigma_{j}$ defined by

$$
\begin{aligned}
f\left(y_{j}, z_{j}\right) & =Y_{j}(2 \pi) \\
& =\exp \left(2 \pi \sqrt{-1}\left[\begin{array}{cc}
\alpha_{j}^{\prime} & 0 \\
0 & \alpha_{j}^{\prime \prime}
\end{array}\right]\right) \cdot\left[\begin{array}{c}
y_{j} \\
z_{j}
\end{array}\right] \\
& =\left[\begin{array}{cc}
\exp \left(2 \pi \sqrt{-1} \alpha_{j}^{\prime}\right) & 0 \\
0 & \exp \left(2 \pi \sqrt{-1} \alpha_{j}^{\prime \prime}\right)
\end{array}\right] \cdot\left[\begin{array}{c}
y_{j} \\
z_{j}
\end{array}\right] \\
& =\left(\exp \left(2 \pi \sqrt{-1} \alpha_{j}^{\prime}\right) y_{j}, \exp \left(2 \pi \sqrt{-1} \alpha_{j}^{\prime \prime}\right) z_{j}\right) \\
& =\left(\lambda_{j}^{\prime} y_{j}, \lambda_{j}^{\prime \prime} z_{j}\right)
\end{aligned}
$$

and this proves the claim.

- In case (e), we consider the foliation $\mathcal{F}_{j}$ on $D_{j} \times \mathbb{C}^{2}$ given by

$$
X_{j}\left(x, y_{j}, z_{j}\right)=\left(x-x_{j}^{0}\right) \frac{\partial}{\partial x}+\frac{\nu}{2 \pi \sqrt{-1}} z_{j} \frac{\partial}{\partial y_{j}} .
$$

Claim 3.2. The holonomy transformation of $\mathcal{F}_{j}$ associated to $\Sigma_{j}$ and $\gamma_{j}$ is of the following form $\left(y_{j}, z_{j}\right) \mapsto\left(y_{j}+\nu z_{j}, z_{j}\right)$, where the vector field $X_{j}$ is given by equation (3.2).

In fact, suppose $\gamma_{j}(\theta)=\left(x_{j}^{0}+\rho_{j} \exp (\sqrt{-1} \theta), 0,0\right) ; 0 \leq \theta \leq 2 \pi$ is a curve where $0<\rho_{j}<\rho$. We consider $\pi_{1}: D_{j} \times \mathbb{C}^{2} \rightarrow D_{j}, \pi_{1}(x, y, z)=x$. Notice that the fibers $\pi_{1}^{-1}(x), x \neq x_{j}^{0}$ are transverse to $\mathcal{F}_{j}$. Let $\Sigma_{j}=\left\{x_{j}^{0}+\rho_{j}\right\} \times \mathbb{C}^{2}$ be a local transverse section and $q=\left(x_{j}^{0}+\rho_{j}, y_{j}, z_{j}\right) \in \Sigma_{j}$. Let $\gamma_{q}(\theta)=\left(x(\theta), y_{j}(\theta), z_{j}(\theta)\right)$ be the lifting of $\gamma_{j}$ by $\pi_{1}$ with base point $q$. Therefore $x^{\prime}(\theta)=\pi_{1}\left(\gamma_{q}^{\prime}(\theta)\right)=\pi_{1}\left(\gamma_{j}^{\prime}(\theta)\right)=$ $\sqrt{-1} \rho_{j} \exp (\sqrt{-1} \theta)$, and, if $Y_{j}=\left(y_{j}, z_{j}\right) \in \mathbb{C}^{2}$, then

$$
\frac{Y_{j}^{\prime}}{x^{\prime}}=\frac{Y_{j}^{\prime}}{\sqrt{-1} \rho_{j} \exp (\sqrt{-1} \theta)} .
$$

On the other hand, by equation (3.2) we have $\frac{d x}{d T}=x-x_{j}^{0}$ and

$$
\frac{d Y_{j}}{d T}=\left[\begin{array}{cc}
0 & \frac{\nu}{2 \pi \sqrt{-1}} \\
0 & 0
\end{array}\right] \cdot\left[\begin{array}{l}
y_{j} \\
z_{j}
\end{array}\right]=A Y_{j},
$$

so we obtain

$$
\frac{d Y_{j}}{d x}=\frac{d Y_{j}}{d T} \cdot \frac{d T}{d x}=\frac{\frac{d Y_{j}}{d T}}{\frac{d x}{d T}}=\frac{A Y_{j}}{x-x_{j}^{0}}
$$

and we have

$$
\frac{Y_{j}^{\prime}}{\sqrt{-1} \rho_{j} \exp (\sqrt{-1} \theta)}=\frac{Y_{j}^{\prime}}{x^{\prime}}=\frac{A Y_{j}}{\rho_{j} \exp (\sqrt{-1} \theta)}
$$


therefore $Y_{j}^{\prime}=\sqrt{-1} A Y_{j}$. Observe that the solution of $Y_{j}^{\prime}=\sqrt{-1} A Y_{j}, Y_{j}(0)=$ $\left(y_{j}, z_{j}\right)$ is $Y_{j}(\theta)=\exp (\sqrt{-1} \theta A) \cdot Y_{j}(0)$. Therefore the holonomy is the biholomorphism $f: \Sigma_{j} \rightarrow \Sigma_{j}$ defined by

$$
\begin{aligned}
f\left(y_{j}, z_{j}\right) & =Y_{j}(2 \pi) \\
& =\exp \left(2 \pi \sqrt{-1}\left[\begin{array}{cc}
0 & \frac{\nu}{2 \pi \sqrt{-1}} \\
0 & 0
\end{array}\right]\right) \cdot\left[\begin{array}{l}
y_{j} \\
z_{j}
\end{array}\right] \\
& =\exp \left(\left[\begin{array}{ll}
0 & \nu \\
0 & 0
\end{array}\right]\right) \cdot\left[\begin{array}{l}
y_{j} \\
z_{j}
\end{array}\right] \\
& =\left(\left[\begin{array}{ll}
1 & 0 \\
0 & 1
\end{array}\right]+\left[\begin{array}{ll}
0 & \nu \\
0 & 0
\end{array}\right]\right) \cdot\left[\begin{array}{l}
y_{j} \\
z_{j}
\end{array}\right] \\
& =\left[\begin{array}{ll}
1 & \nu \\
0 & 1
\end{array}\right] \cdot\left[\begin{array}{l}
y_{j} \\
z_{j}
\end{array}\right] \\
& =\left(y_{j}+\nu z_{j}, z_{j}\right)
\end{aligned}
$$

and this proves the claim.

- In case (b), we consider the singular foliation $\mathcal{F}_{j}$ on $D_{j} \times \mathbb{C}^{2}$ given by

$$
X_{j}\left(x, y_{j}, z_{j}\right)=\left(x-x_{j}^{0}\right) \frac{\partial}{\partial x}+\left(\lambda y_{j}+\frac{\nu}{2 \pi \sqrt{-1} \mu} z_{j}\right) \frac{\partial}{\partial y_{j}}+\lambda z_{j} \frac{\partial}{\partial z_{j}}
$$

where $\exp (2 \pi \sqrt{-1} \lambda)=\mu$.

Let $\gamma_{j}(\theta)=\left(x_{j}^{0}+\rho_{j} \exp (\sqrt{-1} \theta), 0,0\right), 0 \leq \theta \leq 2 \pi$ be a curve where $0<\rho_{j}<\rho$.

Claim 3.3. The holonomy transformation of $\mathcal{F}_{j}$ associated to $\Sigma_{j}$ and $\gamma_{j}$ is of the form $\left(y_{j}, z_{j}\right) \mapsto\left(\mu y_{j}+\nu z_{j}, \mu z_{j}\right)$ where $\mathcal{F}_{j}$ is induced by $X_{j}$ given by (3.3).

In fact, let $\Sigma_{j}=\left\{x_{j}^{0}+\rho_{j}\right\} \times \mathbb{C}^{2}$ be a local transverse section and let $p_{j}=$ $\left(x_{j}^{0}+\rho_{j}, 0,0\right) \in \Sigma_{j}$. Suppose $\pi_{1}: D_{j} \times \mathbb{C}^{2} \rightarrow D_{j}, \pi_{1}(x, y, z)=x$. Notice that the fibers $\pi_{1}^{-1}(x), x \neq x_{j}^{0}$ are transverse to $\mathcal{F}_{j}$. Let $q=\left(x_{j}^{0}+\rho_{j}, y_{j}, z_{j}\right) \in \Sigma_{j}$ and let $\gamma_{q}(\theta)=\left(x(\theta), y_{j}(\theta), z_{j}(\theta)\right)$ be the lifting of $\gamma_{j}$ by $\pi_{1}$ with base point $q$. Therefore $x^{\prime}(\theta)=\pi_{1}\left(\gamma_{q}^{\prime}(\theta)\right)=\pi_{1}\left(\gamma_{j}^{\prime}(\theta)\right)=\sqrt{-1} \rho_{j} \exp (\sqrt{-1} \theta)$, and, if $Y_{j}=\left(y_{j}, z_{j}\right) \in \mathbb{C}^{2}$, then $\frac{Y_{j}^{\prime}}{x^{\prime}}=\frac{Y_{j}^{\prime}}{\sqrt{-1} \rho_{j} \exp (\sqrt{-1} \theta)}$. On the other hand, by equation (3.3) we have $\frac{d x}{d T}=x-x_{j}^{0}$ and

$$
\frac{d Y_{j}}{d T}=\left[\begin{array}{cc}
\lambda & \frac{\nu}{2 \pi \sqrt{-1} \mu} \\
0 & \lambda
\end{array}\right] \cdot\left[\begin{array}{c}
y_{j} \\
z_{j}
\end{array}\right]=A Y_{j}
$$

so we obtain

$$
\frac{d Y_{j}}{d x}=\frac{d Y_{j}}{d T} \cdot \frac{d T}{d x}=\frac{\frac{d Y_{j}}{d T}}{\frac{d x}{d T}}=\frac{A Y_{j}}{x-x_{j}^{0}}
$$

and we have

$$
\frac{Y_{j}^{\prime}}{\sqrt{-1} \rho_{j} \exp (\sqrt{-1} \theta)}=\frac{Y_{j}^{\prime}}{x^{\prime}}=\frac{A Y_{j}}{\rho_{j} \exp (\sqrt{-1} \theta)}
$$

therefore $Y_{j}^{\prime}=\sqrt{-1} A Y_{j}$. Observe that the solution of $Y_{j}^{\prime}=\sqrt{-1} A Y_{j}$ such that $Y_{j}(0)=\left(y_{j}, z_{j}\right)$ is $Y_{j}(\theta)=\exp (\sqrt{-1} \theta A) \cdot Y_{j}(0)$. Therefore the holonomy is the 
biholomorphism $f: \Sigma_{j} \rightarrow \Sigma_{j}$ defined by

$$
\begin{gathered}
f\left(y_{j}, z_{j}\right)=Y_{j}(2 \pi)=\exp \left\{2 \pi \sqrt{-1}\left(\begin{array}{cc}
\lambda & \frac{\nu}{2 \pi \sqrt{-1} \mu} \\
0 & \lambda
\end{array}\right)\right\} \cdot\left(\begin{array}{l}
y_{j} \\
z_{j}
\end{array}\right) \\
=\exp \left\{\left(\begin{array}{cc}
2 \pi \sqrt{-1} \lambda & 0 \\
0 & 2 \pi \sqrt{-1} \lambda
\end{array}\right)+\left(\begin{array}{cc}
0 & \frac{\nu}{\mu} \\
0 & 0
\end{array}\right)\right\} \cdot\left(\begin{array}{c}
c y_{j} \\
z_{j}
\end{array}\right) \\
=\exp \left\{\left(\begin{array}{cc}
2 \pi \sqrt{-1} \lambda & 0 \\
0 & 2 \pi \sqrt{-1} \lambda
\end{array}\right)\right\} \cdot \exp \left\{\left(\begin{array}{cc}
0 & \frac{\nu}{\mu} \\
0 & 0
\end{array}\right)\right\}\left(\begin{array}{c}
y_{j} \\
z_{j}
\end{array}\right) \\
=\left(\begin{array}{cc}
\mu & 0 \\
0 & \mu
\end{array}\right)\left(\begin{array}{cc}
1 & 0 \\
0 & 1
\end{array}\right)+\frac{\nu}{\mu}\left(\begin{array}{cc}
0 & 1 \\
0 & 0
\end{array}\right)\left(\begin{array}{c}
c y_{j} \\
z_{j}
\end{array}\right)=\left(\begin{array}{cc}
\mu & 0 \\
0 & \mu
\end{array}\right)\left(\begin{array}{cc}
1 & \frac{\nu}{\mu} \\
0 & 1
\end{array}\right)\left(\begin{array}{l}
y_{j} \\
z_{j}
\end{array}\right) \\
=\left(\begin{array}{cc}
\mu & \nu \\
0 & \mu
\end{array}\right) \cdot\left(\begin{array}{c}
c y_{j} \\
z_{j}
\end{array}\right)=\left(\mu y_{j}+\nu z_{j}, \mu z_{j}\right)
\end{gathered}
$$

and this proves the claim.

- In case (a), we consider the foliation $\mathcal{F}_{j}$ on $D_{j} \times \mathbb{C}^{2}$ given by

$$
X_{j}\left(x, y_{j}, z_{j}\right)=\left(x-x_{j}^{0}\right) \frac{\partial}{\partial x}+\left(\frac{\mu}{2 \pi \sqrt{-1}} z_{j}-\frac{\mu^{2}}{4 \pi \sqrt{-1}}\right) \frac{\partial}{\partial y_{j}}+\frac{\mu}{2 \pi \sqrt{-1}} \frac{\partial}{\partial z_{j}} .
$$

Claim 3.4. The holonomy transformation of $\mathcal{F}_{j}$ associated to $\Sigma_{j}$ and $\gamma_{j}$ is of the form $\left(y_{j}, z_{j}\right) \mapsto\left(y_{j}+\mu z_{j}, z_{j}+\mu\right)$.

In fact, let $\gamma_{j}(\theta)=\left(x_{j}^{0}+\rho_{j} \exp (\sqrt{-1} \theta), 0,0\right), 0 \leq \theta \leq 2 \pi$ be a curve where $0<\rho_{j}<\rho$, let $\Sigma_{j}=\left\{x_{j}^{0}+\rho_{j}\right\} \times \mathbb{C}^{2}$ be a local transverse section and let $p_{j}=$ $\left(x_{j}^{0}+\rho_{j}, 0,0\right) \in \Sigma_{j}$. Suppose $\pi_{1}: D_{j} \times \mathbb{C}^{2} \rightarrow D_{j}, \pi_{1}(x, y, z)=x$. Notice that the fibers $\pi_{1}^{-1}(x), x \neq x_{j}^{0}$ are transverse to $\mathcal{F}_{j}$. Let $q=\left(x_{j}^{0}+\rho_{j}, y_{j}, z_{j}\right) \in \Sigma_{j}$ and let $\gamma_{q}(\theta)=\left(x(\theta), y_{j}(\theta), z_{j}(\theta)\right)$ be the lifting of $\gamma_{j}$ by $\pi_{1}$ with base point $q$. Therefore $x^{\prime}(\theta)=\pi_{1}\left(\gamma_{q}^{\prime}(\theta)\right)=\pi_{1}\left(\gamma_{j}^{\prime}(\theta)\right)=\sqrt{-1} \rho_{j} \exp (\sqrt{-1} \theta)$, and, if $Y_{j}=\left(y_{j}, z_{j}\right) \in \mathbb{C}^{2}$, then $\frac{Y_{j}^{\prime}}{x^{\prime}}=\frac{Y_{j}^{\prime}}{\sqrt{-1} \rho_{j} \exp (\sqrt{-1} \theta)}$. On the other hand, by equation (3.4) we have $\frac{d x}{d T}=x-x_{j}^{0}$ and

$$
\frac{d Y_{j}}{d T}=\left[\left(\begin{array}{cc}
0 & \frac{\mu}{2 \pi \sqrt{-1}} \\
0 & 0
\end{array}\right)\right] \cdot\left[\left(\begin{array}{c}
c y_{j} \\
z_{j}
\end{array}\right)\right]+\left[\left(\begin{array}{c}
c-\frac{\mu^{2}}{4 \pi \sqrt{-1}} \\
\frac{\mu}{2 \pi \sqrt{-1}}
\end{array}\right)\right]=A Y_{j}+B,
$$

so we obtain

$$
\frac{d Y_{j}}{d x}=\frac{d Y_{j}}{d T} \cdot \frac{d T}{d x}=\frac{\frac{d Y_{j}}{d T}}{\frac{d x}{d T}}=\frac{A Y_{j}+B}{x-x_{j}^{0}}
$$

and we have

$$
\frac{Y_{j}^{\prime}}{\sqrt{-1} \rho_{j} \exp (\sqrt{-1} \theta)}=\frac{Y_{j}^{\prime}}{x^{\prime}}=\frac{A Y_{j}+B}{\rho_{j} \exp (\sqrt{-1} \theta)}
$$

therefore $Y_{j}^{\prime}=\sqrt{-1} A Y_{j}+\sqrt{-1} B$. Observe that the solution of

$$
Y_{j}^{\prime}=\sqrt{-1} A Y_{j}+\sqrt{-1} B
$$

with $Y_{j}(0)=\left(y_{j}, z_{j}\right)$ is

$$
Y_{j}(\theta)=\exp (\sqrt{-1} \theta A) \cdot\left[Y_{j}(0)+\int_{0}^{\theta} \exp (\sqrt{-1} s A) \cdot B(s) d s\right],
$$

that is,

$$
Y_{j}(\theta)=\left(-\frac{\mu^{2}}{4 \pi} \theta-\frac{1}{2} \frac{\mu^{2}}{4 \pi^{2}} \theta^{2}+y_{j}+\frac{\mu^{2}}{4 \pi^{2}} \theta^{2}+\frac{\mu}{2 \pi} \theta z_{j}, \frac{\mu}{2 \pi} \theta+z_{j}\right) .
$$


Therefore the holonomy is the biholomorphism $f: \Sigma_{j} \rightarrow \Sigma_{j}$ defined by

$$
f\left(y_{j}, z_{j}\right)=Y_{j}(2 \pi)=\left(y_{j}+\mu z_{j}, z_{j}+\mu\right)
$$

and this proves the claim.

Let us glue together the foliation on $A_{j} \times \mathbb{C}^{2}$ and the foliations on $D_{j} \times \mathbb{C}^{2}$. First we consider the coordinate systems $\left(x, y_{j}, z_{j}\right) \in D_{j} \times \mathbb{C} P(2)$ and $\left(x, \widetilde{y}_{j}, \widetilde{z}_{j}\right) \in$ $A_{j} \times \mathbb{C} P(2)$. Suppose $f_{j}$ is of type P3 or SL1; then we are in case (c) or case (d). Observe that $A_{j} \cap D_{j}$ is simply connected and $x_{j}^{0} \notin A_{j} \cap D_{j}$, and then we consider the coordinate system $\left(x, \bar{y}_{j}, \bar{z}_{j}\right)$ in $\left(A_{j} \cap D_{j}\right) \times \mathbb{C}^{2}$ where

$$
\bar{y}_{j}=y_{j} \exp \left(-\alpha_{j}^{\prime} \log \left(\frac{x-x_{j}^{0}}{\frac{\rho}{2}}\right)\right), \quad \bar{z}_{j}=z_{j} \exp \left(-\alpha_{j}^{\prime \prime} \log \left(\frac{x-x_{j}^{0}}{\frac{\rho}{2}}\right)\right) .
$$

Here $\log$ is the branch of the logarithm in $\overline{\mathbb{C}} \backslash\{x+\sqrt{-1} y ; x \leq 0\}$ such that $\log (1)=0$. Observe that $x_{j}^{\prime}=x_{j}^{0}+\frac{\rho}{2}$ implies that $\bar{y}_{j}\left(x_{j}^{\prime}, y_{j}\right)=y_{j}, \quad \bar{z}_{j}\left(x_{j}^{\prime}, z_{j}\right)=z_{j}$ and $\bar{y}_{j}(x, 0)=0, \quad \bar{z}_{j}(x, 0)=0$. Then the leaves of the foliation $\mathcal{F}_{j}$ on $D_{j} \times \mathbb{C}^{2}$ restricted to $\left(A_{j} \cap D_{j}\right) \times \mathbb{C}^{2}$ are $\left(\bar{y}_{j}, \bar{z}_{j}\right)=$ constant. Let us identify the point $\left(x, \widetilde{y}_{j}, \widetilde{z}_{j}\right) \in\left(A_{j} \cap D_{j}\right) \times \mathbb{C}^{2} \subset A_{j} \times \mathbb{C}^{2}$ with the point $\left(x, y_{j}, z_{j}\right) \in\left(A_{j} \cap D_{j}\right) \times \mathbb{C}^{2} \subset$ $D_{j} \times \mathbb{C}^{2}$, where

$$
y_{j}=\widetilde{y}_{j} \exp \left(\alpha_{j}^{\prime} \log \left(\frac{x-x_{j}^{0}}{\frac{\rho}{2}}\right)\right), \quad z_{j}=\widetilde{z}_{j} \exp \left(\alpha_{j}^{\prime \prime} \log \left(\frac{x-x_{j}^{0}}{\frac{\rho}{2}}\right)\right) .
$$

Notice that (3.6) is equivalent to identifying $\left(x, \widetilde{y}_{j}, \widetilde{z}_{j}\right)$ with $\left(x, y_{j}, z_{j}\right)$ and then, with (3.6) we are gluing together plaques of the horizontal foliation $\widetilde{\mathcal{F}}_{j}$ on $\left(A_{j} \cap\right.$ $\left.D_{j}\right) \times \mathbb{C}^{2} \subset A_{j} \times \mathbb{C}^{2}$ with plaques of $\mathcal{F}_{j}$ on $\left(A_{j} \cap D_{j}\right) \times \mathbb{C}^{2} \subset D_{j} \times \mathbb{C}^{2}$. Observe that this identification sends the fiber $\{x=c\} \subset A_{j} \times \mathbb{C}^{2}, c \in A_{j} \cap D_{j}$, in the fiber $\{x=c\} \subset D_{j} \times \mathbb{C}^{2}$, and the holonomy of the curve $\beta_{j}=\alpha_{j} * \gamma_{j} * \alpha_{j}^{-1}$ in the section $\Sigma_{j}^{\prime \prime}=\left\{x_{j}^{\prime \prime}\right\} \times \mathbb{C}^{2} \subset A_{j} \times \mathbb{C}^{2}$ with respect to the foliation obtained by gluing together the $\widetilde{\mathcal{F}}_{j}$ and $\mathcal{F}_{j}$ is of the form $\left(\widetilde{y}_{j}, \widetilde{z}_{j}\right) \mapsto\left(\lambda_{j}^{\prime} \widetilde{y}_{j}, \lambda_{j}^{\prime \prime} \widetilde{z}_{j}\right)$. Let us call this foliation $\widetilde{\mathcal{F}}_{j}$ also.

By analogy with these ideas, if $f_{j}$ is of type $P 1 S L 1$, then we are in case (e) and we consider the coordinate system $\left(x, \bar{y}_{j}, \bar{z}_{j}\right)$ in $\left(A_{j} \cap D_{j}\right) \times \mathbb{C}^{2}$ where

$$
\bar{y}_{j}=y_{j}-\frac{\nu}{2 \pi \sqrt{-1}} \log \left(\frac{x-x_{j}}{\frac{\rho}{2}}\right) z_{j}, \quad \bar{z}_{j}=z_{j},
$$

i.e.,

$$
\left(c c l\left[\left(\begin{array}{c}
c \bar{y}_{j} \\
\bar{z}_{j}
\end{array}\right)\right]=\left[\left(\begin{array}{cc}
1 & -\frac{\nu}{2 \pi \sqrt{-1}} \log \left(\frac{x-x_{j}^{0}}{\frac{\rho}{2}}\right) \\
0 & 1
\end{array}\right)\right] \cdot\left[\left(\begin{array}{c}
c y_{j} \\
z_{j}
\end{array}\right)\right]\right) .
$$

The leaves of the foliation $\mathcal{F}_{j}$ on $D_{j} \times \mathbb{C}^{2}$ restricted to $\left(A_{j} \cap D_{j}\right) \times \mathbb{C}^{2}$ are $\left(\bar{y}_{j}, \bar{z}_{j}\right)=$ the constant vector again. We identify the point $\left(x, \widetilde{y}_{j}, \widetilde{z}_{j}\right) \in\left(A_{j} \cap D_{j}\right) \times$ $\mathbb{C}^{2} \subset A_{j} \times \mathbb{C}^{2}$ with the point $\left(x, y_{j}, z_{j}\right) \in\left(A_{j} \cap D_{j}\right) \times \mathbb{C}^{2} \subset D_{j} \times \mathbb{C}^{2}$, where

$$
y_{j}=\widetilde{y}_{j}+\frac{\nu}{2 \pi \sqrt{-1}} \log \left(\frac{x-x_{j}}{\frac{\rho}{2}}\right) \widetilde{z}_{j}, \quad z_{j}=\widetilde{z}_{j}
$$

i.e.,

$$
\left[\begin{array}{l}
\bar{y}_{j} \\
\bar{z}_{j}
\end{array}\right]=\left[\begin{array}{cc}
1 & \frac{\nu}{2 \pi \sqrt{-1}} \log \left(\frac{x-x_{j}^{0}}{\frac{\rho}{2}}\right) \\
0 & 1
\end{array}\right] \cdot\left[\begin{array}{c}
y_{j} \\
z_{j}
\end{array}\right] .
$$

Notice that (3.8) is equivalent to identify $\left(x, \widetilde{y}_{j}, \widetilde{z}_{j}\right)$ with $\left(x, y_{j}, z_{j}\right)$. 
And on the one hand, if $f_{j}$ is of type $\mathrm{P} 2$, we are in case (b) and we change the identifications (3.5) and (3.6) by

$$
\begin{gathered}
\bar{y}_{j}=y_{j} \exp \left(-\lambda \log \left(\frac{x-x_{j}^{0}}{\frac{\rho}{2}}\right)\right)-z_{j} \frac{\nu}{2 \pi \sqrt{-1} \mu} \log \left(\frac{x-x_{j}^{0}}{\frac{\rho}{2}}\right) \exp \left(-\lambda \log \left(\frac{x-x_{j}^{0}}{\frac{\rho}{2}}\right)\right), \\
\bar{z}_{j}=z_{j} \exp \left(-\lambda \log \left(\frac{x-x_{j}^{0}}{\frac{\rho}{2}}\right)\right)
\end{gathered}
$$

i.e.,

$$
\left[\begin{array}{c}
\bar{y}_{j} \\
\bar{z}_{j}
\end{array}\right]=\left[\begin{array}{cc}
\exp \left(-\lambda \log \left(\frac{x-x_{j}^{0}}{\frac{\rho}{2}}\right)\right) & -\exp \left(-\lambda \log \left(\frac{x-x_{j}^{0}}{\frac{\rho}{2}}\right)\right) \frac{\nu}{2 \pi \sqrt{-1}} \log \left(\frac{x-x_{j}^{0}}{\frac{\rho}{2}}\right) \\
0 & \exp \left(-\lambda \log \left(\frac{x-x_{j}^{0}}{\frac{\rho}{2}}\right)\right)
\end{array}\right] \cdot\left[\begin{array}{c}
y_{j} \\
z_{j}
\end{array}\right]
$$

and

$$
\begin{gathered}
y_{j}=\widetilde{y}_{j} \exp \left(\lambda \log \left(\frac{x-x_{j}^{0}}{\frac{\rho}{2}}\right)\right)+z_{j} \frac{\nu}{2 \pi \sqrt{-1} \mu} \log \left(\frac{x-x_{j}^{0}}{\frac{\rho}{2}}\right) \exp \left(\lambda \log \left(\frac{x-x_{j}^{0}}{\frac{\rho}{2}}\right)\right), \\
z_{j}=\widetilde{z}_{j} \exp \left(\lambda \log \left(\frac{x-x_{j}^{0}}{\frac{\rho}{2}}\right)\right)
\end{gathered}
$$

i.e.,

$$
\left[\begin{array}{l}
y_{j} \\
z_{j}
\end{array}\right]=\left[\begin{array}{cc}
\exp \left(\lambda \log \left(\frac{x-x_{j}^{0}}{\frac{\rho}{2}}\right)\right) & \exp \left(\lambda \log \left(\frac{x-x_{j}^{0}}{\frac{\rho}{2}}\right)\right) \frac{\nu}{2 \pi \sqrt{-1}} \log \left(\frac{x-x_{j}^{0}}{\frac{\rho}{2}}\right) \\
0 & \exp \left(\lambda \log \left(\frac{x-x_{j}^{0}}{\frac{\rho}{2}}\right)\right)
\end{array}\right] \cdot\left[\begin{array}{c}
\widetilde{y}_{j} \\
\widetilde{z}_{j}
\end{array}\right]
$$

and we repeat these ideas. On the other hand, if $f_{j}$ is of type $\mathrm{P} 1$ we are in case (a) and the identifications are

$$
\begin{gathered}
\bar{y}_{j}=y_{j}+\left(z_{j} \frac{\mu}{2 \pi \sqrt{-1}}+\frac{1}{2} \frac{\mu^{2}}{(2 \pi \sqrt{-1})^{2}} \log \left(\frac{x-x_{j}^{0}}{\frac{\rho}{2}}\right)+\frac{\mu^{2}}{4 \pi \sqrt{-1}}\right) \cdot \log \left(\frac{x-x_{j}^{0}}{\frac{\rho}{2}}\right), \\
\bar{z}_{j}=z_{j}+\frac{\mu}{2 \pi \sqrt{-1}} \log \left(\frac{x-x_{j}^{o}}{\frac{\rho}{2}}\right)
\end{gathered}
$$

i.e.,

$$
\begin{aligned}
{\left[\begin{array}{l}
\bar{y}_{j} \\
\bar{z}_{j}
\end{array}\right]=} & {\left[\begin{array}{cc}
1 & -\frac{\mu}{2 \pi \sqrt{-1}} \log \left(\frac{x-x_{j}^{0}}{\frac{\rho}{2}}\right) \\
0 & 1
\end{array}\right] \cdot\left[\begin{array}{c}
y_{j} \\
z_{j}
\end{array}\right] } \\
& +\left[\begin{array}{c}
\left(\frac{1}{2} \frac{\mu^{2}}{(2 \pi \sqrt{-1})^{2}} \log \left(\frac{x-x_{j}^{0}}{\frac{\rho}{2}}\right)+\frac{\mu^{2}}{4 \pi \sqrt{-1}}\right) \cdot \log \left(\frac{x-x_{j}^{0}}{\frac{\rho}{2}}\right) \\
\frac{\mu}{2 \pi \sqrt{-1}} \log \left(\frac{x-x_{j}^{o}}{\frac{\rho}{2}}\right)
\end{array}\right]
\end{aligned}
$$

and

$$
\begin{aligned}
y_{j}=\widetilde{y}_{j}+\left(\widetilde{z}_{j} \frac{\mu}{2 \pi \sqrt{-1}}+\right. & \left.\frac{1}{2} \frac{\mu^{2}}{(2 \pi \sqrt{-1})^{2}} \log \left(\frac{x-x_{j}^{0}}{\frac{\rho}{2}}\right)-\frac{\mu^{2}}{4 \pi \sqrt{-1}}\right) \cdot \log \left(\frac{x-x_{j}^{0}}{\frac{\rho}{2}}\right), \\
z_{j} & =\widetilde{z}_{j}+\frac{\mu}{2 \pi \sqrt{-1}} \log \left(\frac{x-x_{j}^{0}}{\frac{\rho}{2}}\right)
\end{aligned}
$$


i.e.,

$$
\begin{aligned}
{\left[\begin{array}{l}
y_{j} \\
z_{j}
\end{array}\right]=} & {\left[\begin{array}{ll}
1 & \frac{\mu}{2 \pi \sqrt{-1}} \log \left(\frac{x-x_{j}^{0}}{\frac{\rho}{2}}\right) \\
0 & 1
\end{array}\right] \cdot\left[\begin{array}{c}
\widetilde{y}_{j} \\
\widetilde{z}_{j}
\end{array}\right] } \\
& +\left[\begin{array}{c}
\left(\frac{1}{2} \frac{\mu^{2}}{(2 \pi \sqrt{-1})^{2}} \log \left(\frac{x-x_{j}^{0}}{\frac{\rho}{2}}\right)-\frac{\mu^{2}}{4 \pi \sqrt{-1}}\right) \cdot \log \left(\frac{x-x_{j}^{0}}{\frac{\rho}{2}}\right) \\
\frac{\mu}{2 \pi \sqrt{-1}} \log \left(\frac{x-x_{j}^{o}}{\frac{\rho}{2}}\right)
\end{array}\right] .
\end{aligned}
$$

Now let us glue together the new foliation $\widetilde{\mathcal{F}}_{j}$ on $\left(A_{j} \cup D_{j}\right) \times \mathbb{C}^{2}$ with the foliation $\mathcal{F}_{0}$ on $\left(A_{j} \cap D_{0}\right) \times \mathbb{C}^{2}$. First, if $f_{0}$ is of type P3 or SL1, we consider the coordinate system $\left(x, \bar{y}_{0}, \bar{z}_{0}\right)$ in $\left(A_{j} \cap D_{0}\right) \times \mathbb{C}^{2}$ where

$$
\bar{y}_{0}=y_{0} \exp \left(-\alpha_{0}^{\prime} \log \left(\frac{x}{x_{j}^{\prime \prime}}\right)\right), \quad \bar{z}_{0}=z_{0} \exp \left(-\alpha_{0}^{\prime \prime} \log \left(\frac{x}{x_{j}^{\prime \prime}}\right)\right) .
$$

Observe that the leaves of the foliation $\widetilde{\mathcal{F}}_{j}$ on $D_{0} \times \mathbb{C}^{2}$ restricted to $\left(A_{j} \cap D_{0}\right) \times \mathbb{C}^{2}$ are $\left(\bar{y}_{0}, \bar{z}_{0}\right)=$ the constant vector. We identify the points $\left(x, \widetilde{y}_{j}, \widetilde{z}_{j}\right) \in\left(A_{j} \cap D_{0}\right) \times \mathbb{C}^{2} \subset$ $A_{j} \times \mathbb{C}^{2}$ with $\left(x, y_{0}, z_{0}\right) \in\left(A_{j} \cap D_{0}\right) \times \mathbb{C}^{2}$ by

$$
y_{0}=\widetilde{y}_{j} \exp \left(\alpha_{0}^{\prime} \log \left(\frac{x}{x_{j}^{\prime \prime}}\right)\right), \quad z_{0}=\widetilde{z}_{j} \exp \left(\alpha_{0}^{\prime \prime} \log \left(\frac{x}{x_{j}^{\prime \prime}}\right)\right)
$$

where $\lambda_{0}^{\prime}=\exp \left(2 \pi \sqrt{-1} \alpha_{0}^{\prime}\right)$ and $\lambda_{0}^{\prime \prime}=\exp \left(2 \pi \sqrt{-1} \alpha_{0}^{\prime \prime}\right)$.

Notice that identification (3.18) glues together plaques of $\widetilde{\mathcal{F}}_{j}$ on $\left(A_{j} \cup D_{j}\right) \times \mathbb{C}^{2}$ with plaques of $\mathcal{F}_{0}$ and this defines a new foliation such that the holonomy of the curve $\beta_{j}$ in the section $\left\{x_{j}^{\prime \prime}\right\} \times \mathbb{C}^{2} \subset D_{0} \times \mathbb{C}^{2}$ is given by $\left(y_{0}, z_{0}\right) \mapsto\left(\lambda_{j}^{\prime} y_{0}, \lambda_{j}^{\prime \prime} z_{0}\right)$.

By analogy with this case, if $f_{0}$ is of type $P 1 S L 1$, we consider the coordinate system $\left(x, \bar{y}_{0}, \bar{z}_{0}\right)$ in $\left(A_{j} \cap D_{0}\right) \times \mathbb{C}^{2}$ where

$$
\bar{y}_{0}=y_{0}-\frac{\nu}{2 \pi \sqrt{-1}} \log \left(\frac{x}{x_{j}^{\prime \prime}}\right) z_{0}, \quad \bar{z}_{0}=z_{0} .
$$

We identify the point $\left(x, \widetilde{y}_{j}, \widetilde{z}_{j}\right) \in\left(A_{j} \cap D_{0}\right) \times \mathbb{C}^{2} \subset A_{j} \times \mathbb{C}^{2}$ with the point $\left(x, y_{0}, z_{0}\right) \in\left(A_{j} \cap D_{0}\right) \times \mathbb{C}^{2} \subset D_{0} \times \mathbb{C}^{2}$ by

$$
y_{0}=\widetilde{y}_{j}+\frac{\nu}{2 \pi \sqrt{-1}} \log \left(\frac{x}{x_{j}^{\prime \prime}}\right) \widetilde{z}_{j}, \quad z_{0}=\widetilde{z}_{j},
$$

However, if $f_{0}$ is of type $\mathrm{P} 2$, then we consider the coordinate system $\left(x, \bar{y}_{0}, \bar{z}_{0}\right)$ in $\left(A_{j} \cap D_{0}\right) \times \mathbb{C}^{2}$ where

$$
\begin{gathered}
\bar{y}_{0}=y_{0} \exp \left(-\lambda \log \left(\frac{x}{x_{j}^{\prime \prime}}\right)\right)-z_{0} \frac{\nu}{2 \pi \sqrt{-1} \mu} \log \left(\frac{x}{x_{j}^{\prime \prime}}\right) \exp \left(-\lambda \log \left(\frac{x}{x_{j}^{\prime \prime}}\right)\right), \\
\bar{z}_{0}=z_{0} \exp \left(-\lambda \log \left(\frac{x}{x_{j}^{\prime \prime}}\right)\right) .
\end{gathered}
$$

We identify the point $\left(x, \widetilde{y}_{j}, \widetilde{z}_{j}\right) \in\left(A_{j} \cap D_{0}\right) \times \mathbb{C}^{2} \subset A_{j} \times \mathbb{C}^{2}$ with the point $\left(x, y_{0}, z_{0}\right) \in\left(A_{j} \cap D_{0}\right) \times \mathbb{C}^{2}$ by

$$
\begin{gathered}
y_{0}=\widetilde{y}_{j} \exp \left(\lambda \log \left(\frac{x}{x_{j}^{\prime \prime}}\right)\right)+\widetilde{z}_{j} \frac{\nu}{2 \pi \sqrt{-1} \mu} \log \left(\frac{x}{x_{j}^{\prime \prime}}\right) \exp \left(\lambda \log \left(\frac{x}{x_{j}^{\prime \prime}}\right)\right), \\
z_{0}=\widetilde{z}_{j} \exp \left(\lambda \log \left(\frac{x}{x_{j}^{\prime \prime}}\right)\right) .
\end{gathered}
$$


On the other hand, if $f_{0}$ is of type $\mathrm{P} 1$, then we consider the coordinate system $\left(x, \bar{y}_{0}, \bar{z}_{0}\right)$ in $\left(A_{j} \cap D_{0}\right) \times \mathbb{C}^{2}$ where

$$
\begin{gathered}
\bar{y}_{0}=y_{0}+\left(z_{0} \frac{\mu}{2 \pi \sqrt{-1}}+\frac{1}{2} \frac{\mu^{2}}{(2 \pi \sqrt{-1})^{2}} \log \left(\frac{x}{x_{j}^{\prime \prime}}\right)+\frac{\mu^{2}}{4 \pi \sqrt{-1}}\right) \cdot \log \left(\frac{x}{x_{j}^{\prime \prime}}\right), \\
\bar{z}_{0}=z_{0}+\frac{\mu}{2 \pi \sqrt{-1}} \log \left(\frac{x}{x_{j}^{\prime \prime}}\right),
\end{gathered}
$$

and we identify the point $\left(x, \widetilde{y}_{j}, \widetilde{z}_{j}\right) \in\left(A_{j} \cap D_{0}\right) \times \mathbb{C}^{2} \subset A_{j} \times \mathbb{C}^{2}$ with the point $\left(x, y_{0}, z_{0}\right) \in\left(A_{j} \cap D_{0}\right) \times \mathbb{C}^{2}$ by

$$
\begin{gathered}
y_{0}=\widetilde{y}_{j}+\left(\widetilde{z}_{j} \frac{\mu}{2 \pi \sqrt{-1}}+\frac{1}{2} \frac{\mu^{2}}{(2 \pi \sqrt{-1})^{2}} \log \left(\frac{x}{x_{j}^{\prime \prime}}\right)-\frac{\mu^{2}}{4 \pi \sqrt{-1}}\right) \cdot \log \left(\frac{x}{x_{j}^{\prime \prime}}\right), \\
z_{0}=\widetilde{z}_{j}+\frac{\mu}{2 \pi \sqrt{-1}} \log \left(\frac{x}{x_{j}^{\prime \prime}}\right) .
\end{gathered}
$$

Now suppose $\gamma_{0}(\theta)=\frac{\rho}{2} \exp (\sqrt{-1} \theta), 0 \leq \theta \leq 2 \pi$, and for every $j=1, \ldots, k$ let $\mu_{j}$ be the segment of $\gamma_{0}$ between $x_{j}^{\prime \prime}$ and $\frac{\rho}{2}$ in the positive sense. Let

$$
\delta_{j}=\mu_{j} * \beta_{j} * \mu_{j}^{-1}=\mu_{j} * \alpha_{j} * \gamma_{j} * \alpha_{j}^{-1} * \mu_{j}^{-1},
$$

where $\gamma_{j}(\theta)=\frac{\rho}{2} \exp (\sqrt{-1} \theta)+x_{j}, \theta \in[0,2 \pi]$ and $\Sigma_{0}=\left\{\frac{\rho}{2}\right\} \times \mathbb{C}^{2}$. The holonomy of the curve $\delta_{j}$ in $\Sigma_{0}$ is given by conditions in item (3).

Notice that we have a foliation on $\left(\bigcup A_{k} \cup \bigcup D_{j}\right) \times \mathbb{C} P(2)$ such that the holonomy of the curve $\beta_{j}$ is a biholomorphism of $\mathbb{C} P(2)$.

Now let $\widetilde{M}$ be a complex manifold obtained by gluing together all the foliations $\widetilde{\mathcal{F}}_{j}$. Let $\widetilde{\mathcal{F}}$ be the foliation in $\widetilde{M}$ obtained at the end of the process. Then the holonomy of the leaf $U=\left(\bigcup_{i=0}^{r} A_{i}\right) \cup\left(\bigcup_{j=0}^{r} D_{j}\right)$ in $\Sigma_{0}$ is generated by $f_{1}, \ldots, f_{r}$. Notice that $\widetilde{M}$ admits the vertical foliation $\{x=$ constant $\}$ and it cuts $U$ at a single point; hence, we can define a projection $\widetilde{p}: \widetilde{M} \rightarrow U$ such that $\widetilde{p}^{-1}(x)$ is the leaf of this new foliation. Observe that this new foliation is transverse to $\widetilde{\mathcal{F}}$ in $\widetilde{M} \backslash\left\{x=x_{0}^{0}, \ldots, x=x_{r}^{0}\right\}$.

Now let us glue together the foliation $\widetilde{\mathcal{F}}$ and the foliation on $V \times \mathbb{C} P(2)$. First, we consider the annulus $A=T \cap U$, if $\delta$ is a closed curve in $A$ which generates the homotopy of $A$, then the holonomy of $\delta$ with respect to $\widetilde{\mathcal{F}}$ in some transversal section is trivial, because $\delta$ is homotopic to the curve $\delta_{1} * \cdots * \delta_{r} * \gamma_{0}$ in $U \backslash \bigcup_{j=0}^{k}\left\{x_{j}^{0}\right\}$ and the holonomy of this is the identity. Then we obtain that the restricted foliation $\left.\widetilde{\mathcal{F}}\right|_{\tilde{p}^{-1}(A)}$ is a product foliation; that is, there is a biholomorphism $\varphi$ of the same neighborhood $W$ of $\widetilde{p}^{-1}(A)$ onto $A \times \Delta$, where $\Delta \subset \mathbb{C}^{2}$ is a polydisc such that $\varphi$ sends leaves of $\left.\widetilde{\mathcal{F}}\right|_{W}$ onto leaves of trivial foliation. This map $\varphi$ can be chosen such that $\varphi\left(p^{-1}(x) \cap W\right)=\{x\} \times \Delta$. In order to complete the construction of a complex 3 -manifold $M^{3}$ in the conditions of the theorem it is sufficient to glue together the foliation $\widetilde{\mathcal{F}}$ on $\widetilde{M}$ and the trivial foliation on $V \times \mathbb{C} P(2)$ by $\varphi$. This proves the first part of the statement.

Notice that if we assume that $f_{j}(j=1, \ldots, r)$ and $f_{0}$ are all of type P3 or SL1, then the vector field $X_{j}$ in the affine coordinate $\left(x, u_{j}=\frac{1}{y_{j}}, v_{j}=\frac{z_{j}}{y_{j}}\right) \in D_{j} \times \mathbb{C}^{2}$ is given by

$$
X_{j}\left(x, u_{j}, v_{j}\right)=\left(x-x_{j}^{0}\right) \frac{\partial}{\partial x}-\alpha_{j}^{\prime} u_{j} \frac{\partial}{\partial u_{j}}+\left(\alpha_{j}^{\prime \prime}-\alpha_{j}^{\prime}\right) v_{j} \frac{\partial}{\partial v_{j}}
$$


and, in affine coordinates $\left(x, s_{j}=\frac{y_{j}}{z_{j}}, t_{j}=\frac{1}{z_{j}}\right)$,

$$
X_{j}\left(x, s_{j}, t_{j}\right)=\left(x-x_{j}^{0}\right) \frac{\partial}{\partial x}+\left(\alpha_{j}^{\prime}-\alpha_{j}^{\prime \prime}\right) s_{j} \frac{\partial}{\partial s_{j}}-\alpha_{j}^{\prime \prime} t_{j} \frac{\partial}{\partial t_{j}} .
$$

We take the coordinate systems $\left(x, \bar{u}_{j}, \bar{v}_{j}\right)$ and $\left(x, \bar{s}_{j}, \bar{t}_{j}\right)$ in $\left(A_{j} \cap D_{j}\right) \times \mathbb{C}^{2}$ where

$$
\begin{gathered}
\bar{u}_{j}=u_{j} \exp \left(\alpha_{j}^{\prime} \log \left(\frac{x-x_{j}^{0}}{\frac{\rho}{2}}\right)\right), \quad \bar{v}_{j}=v_{j} \exp \left(-\left(\alpha_{j}^{\prime \prime}-\alpha_{j}^{\prime}\right) \log \left(\frac{x-x_{j}^{0}}{\frac{\rho}{2}}\right)\right), \\
\bar{s}_{j}=s_{j} \exp \left(-\left(\alpha_{j}^{\prime}-\alpha_{j}^{\prime \prime}\right) \log \left(\frac{x-x_{j}^{0}}{\frac{\rho}{2}}\right)\right), \quad \bar{t}_{j}=t_{j} \exp \left(\alpha_{j}^{\prime \prime} \log \left(\frac{x-x_{j}^{0}}{\frac{\rho}{2}}\right)\right) .
\end{gathered}
$$

We identify the points $\left(x, \widetilde{u}_{j}, \widetilde{v}_{j}\right)$ and $\left(x, \widetilde{s}_{j}, \widetilde{t}_{j}\right)$ in $\left(A_{j} \cap D_{j}\right) \times \mathbb{C}^{2} \subset A_{j} \times \mathbb{C}^{2}$ with the points $\left(x, u_{j}, v_{j}\right)$ and $\left(x, s_{j}, t_{j}\right)$ in $\left(A_{j} \cap D_{j}\right) \times \mathbb{C}^{2} \subset D_{j} \times \mathbb{C}^{2}$, respectively, where

$$
\begin{aligned}
& u_{j}=\widetilde{u}_{j} \exp \left(-\alpha_{j}^{\prime} \log \left(\frac{x-x_{j}^{0}}{\frac{\rho}{2}}\right)\right), \quad v_{j}=\widetilde{v}_{j} \exp \left(\left(\alpha_{j}^{\prime \prime}-\alpha_{j}^{\prime}\right) \log \left(\frac{x-x_{j}^{0}}{\frac{\rho}{2}}\right)\right), \\
& s_{j}=\widetilde{s}_{j} \exp \left(\left(\alpha_{j}^{\prime}-\alpha_{j}^{\prime \prime}\right) \log \left(\frac{x-x_{j}^{0}}{\frac{\rho}{2}}\right)\right), \quad t_{j}=\widetilde{t}_{j} \exp \left(-\alpha_{j}^{\prime \prime} \log \left(\frac{x-x_{j}^{0}}{\frac{\rho}{2}}\right)\right) .
\end{aligned}
$$

When we take the affine coordinates $\left(x, u_{0}=\frac{1}{y_{0}}, v_{0}=\frac{z_{0}}{y_{0}}\right),\left(x, s_{0}=\frac{y_{0}}{z_{0}}, t_{0}=\frac{1}{z_{0}}\right) \in$ $D_{0} \times \mathbb{C}^{2}$, we identify the points $\left(x, \widetilde{u}_{j}, \widetilde{v}_{j}\right),\left(x, \widetilde{s}_{j}, \widetilde{t}_{j}\right) \in\left(A_{j} \cap D_{0}\right) \times \mathbb{C}^{2} \subset A_{j} \cap \mathbb{C}^{2}$ with the points $\left(x, u_{0}, v_{0}\right),\left(x, s_{0}, t_{0}\right) \in\left(A_{j} \cap D_{0}\right) \times \mathbb{C}^{2} \subset D_{0} \times \mathbb{C}^{2}$ respectively, where

$$
\begin{gathered}
u_{0}=\widetilde{u}_{j} \exp \left(-\alpha_{0}^{\prime} \log \left(\frac{x}{x_{j}^{\prime \prime}}\right)\right), \quad v_{0}=\widetilde{v}_{j} \exp \left(\left(\alpha_{0}^{\prime \prime}-\alpha_{0}^{\prime}\right) \log \left(\frac{x}{x_{j}^{\prime \prime}}\right)\right), \\
s_{0}=\widetilde{s}_{j} \exp \left(\left(\alpha_{0}^{\prime}-\alpha_{0}^{\prime \prime}\right) \log \left(\frac{x}{x_{j}^{\prime \prime}}\right)\right), \quad t_{0}=\widetilde{t}_{j} \exp \left(-\alpha_{0}^{\prime \prime} \log \left(\frac{x}{x_{j}^{\prime \prime}}\right)\right) .
\end{gathered}
$$

This proves the existence of a compact three-manifold $M^{3}$ with a bundle structure with fiber $\mathbb{C} P(2)$ over $\mathbb{C} P(1)$, and a (Riccati) holomorphic foliation $\mathcal{F}$ on $M^{3}$, transverse to almost every fiber, with invariant fibers $\pi^{-1}\left(x_{0}\right), \ldots, \pi^{-1}\left(x_{r}\right)$, and having global holonomy conjugate to the group $G=\left\langle f_{1}, \ldots, f_{r}\right\rangle$. It remains to observe that $M^{3}$ is biholomorphic to a projective manifold because of the bundle structure with fiber $\mathbb{C} P(2)$ over $\mathbb{C} P(1)$. Finally, the foliation $\mathcal{F}$ is conjugate to an algebraic foliation on the projective model because any holomorphic foliation on a projective manifold is algebraic.

Question 3.5. Assume that $f_{j}(j=1, \ldots, r)$ and $f_{0}$ are all of type P3 or SL1. Is it true that from the above construction we have $M^{3}=\overline{\mathbb{C}} \times \mathbb{C} P(2)$ and $\eta$ is trivial?

\section{REFERENCES}

[1] Camacho, César; Lins Neto, Alcides, Geometry theory of foliations. Translated from the Portuguese by Sue E. Goodman. Birkhäuser Boston, Inc., Boston, MA, 1985. vi + 205 pp. MR824240 (87a:57029)

[2] Godbillon, Claude, Feuilletages. Études géométriques. With a preface by G. Reeb. Progress in Mathematics, 98. Birkhäuser Verlag, Basel, 1991. MR 1120547 (93i:57038)

[3] Griffiths, Phillip; Harris, Joseph, Principles of algebraic geometry. Reprint of the 1978 original. Wiley Classics Library. John Wiley \& Sons, Inc., New York, 1994. xiv + 813 pp. MR.1288523 (95d:14001)

[4] Gunning, Robert C., Introduction to holomorphic functions of several variables. Vol. I. Function theory. The Wadsworth \& Brooks/Cole Mathematics Series. Wadsworth \& Brooks/Cole Advanced Books \& Software, Pacific Grove, CA, 1990. xx + 203 pp. MR.1052649(92b:32001a) 
[5] Lins Neto, Alcides, Construction of singular holomorphic vector fields and foliations in dimension two. J. Differential Geom. 26 (1987), no. 1, 1 - 31. MR892029 (88f:32047)

[6] Pan, I.; Sebastiani, M., Les Équations Différentielles Algébriques et les Singularités Mobiles. Monografias de Matemática do IMPA. Rio de Janeiro, Brazil, 2005. MR2101092 (2006f:32042b)

[7] Pan, I.; Sebastiani, M., Sur les équations différentielles algébriques admettant des solutions avec une singularité essentielle. Ann. Inst. Fourier (Grenoble) 51 (2001), no. 6, 1621-1633. MR.1871283(2002m:34132)

[8] Scárdua, Bruno, On complex codimension-one foliations transverse fibrations. J. Dyn. Control Syst. 11 (2005), no. 4, 575-603. MR2170665 (2006g:32049)

[9] Scárdua, Bruno, Holomorphic foliations transverse to fibrations on hyperbolic manifolds. Complex Variables Theory Appl. 46 (2001), no. 3, 219-240. MR.1869737(2002m:32048)

Departamento de Geometria, Instituto de Matemática, Universidade Federal FlumiNense, Niteroi, Rio de Janeiro 24.020-140, BraziL

E-mail address: fabio@mat.uff.br

Instituto de Matematica, Universidade Federal do Rio de Janeiro, CP. 68530-Rio de JANEIRO-RJ, 21945-970, BRAZIL

E-mail address: scardua@im.ufrj.br 